\title{
Uso de los recursos de cita y referencia en la escritura de la tesis de posgrado y su relación con el plagio textual
}

\section{The use of citation and reference resources in the writing of post- graduate theses and its relationship with textual plagiarism}

DOI: https://doi.org/10.32870/dse.v0i23.952

\author{
María Cristina Castro Azuara* \\ Martín Sánchez Camargo**
}

\begin{abstract}
Resumen
El incremento de casos de plagio en el mundo académico y científico, y la necesidad de que las instituciones formadoras de investigadores garanticen la calidad y originalidad de las investigaciones científicas de sus egresados, nos obliga a poner atención en los procesos de enseñanza de la escritura académicocientífica en el posgrado. En el marco del análisis lingüístico del discurso, en este artículo describimos los recursos de atribución del conocimiento utilizados en el proceso de escritura de tesis, por estudiantes de una maestría en el área de las humanidades. Nuestro propósito es describir, desde un estudio exploratorio, las estrategias que los estudiantes utilizan para generar textos propios a partir del conocimiento disciplinar tomado de otras fuentes. Los resultados muestran que los tesistas presentan problemas al identificar las variadas funciones que las citas y referencias cumplen en la construcción del diálogo académico y científico, situación que los coloca en alto riesgo de cometer plagio. Estos hallazgos nos llevan a abrir la reflexión sobre el tema de la escritura en el posgrado, y a la necesidad de proponer planes de intervención en el marco de la alfabetización académica y científica que, al abordar el plagio como un fenómeno relacionado con el uso del lenguaje, permitan a los egresados de estos programas generar textos propios.
\end{abstract}

Palabras claves: plagio - escritura académica - tesis de grado - atribución del conocimiento.

\begin{abstract}
The increase in cases of plagiarism in the academic and scientific world and the need for universities to guarantee the quality and originality of their graduate's theses force us to pay attention to the processes of teaching academic-scientific writing. Based on the linguistic discourse analysis, we describe the knowledge attribution resources used by masters' degree students in the humanities when writing their theses. Our
\end{abstract}

* Doctora en Ciencias del Lenguaje. Líneas de investigación: escritura e identidad disciplinar; alfabetización académica; escritura académicocientífica y formación de investigadores. Universidad Autónoma de Tlaxcala. México. mariacristina.castro@uatx.mx

** Doctor en Ciencias del Lenguaje. Líneas de investigación: argumentación académica y científica; plagio y escritura académica; procesos de literacidad disciplinar. Universidad de las Américas, Puebla. México. martin.sanchez@udlap.mx 
exploratory study seeks to show the strategies that students use to generate their own texts using disciplinary knowledge taken from other sources. The results show that thesis writers have trouble identifying the different functions that citations and references fulfill in the construction of academic and scientific dialogue, which places them at great risk of committing plagiarism. These findings lead us to reflect on the issue of academic writing in the university, and the need to propose intervention plans that address plagiarism as a phenomenon related to the use of language and allow students in these programs to generate their own texts.

Key words: plagiarism - academic writing - thesis - knowledge attribution.

\section{Introducción}

El problema del plagio y su impacto negativo en la formación académica y en la práctica profesional de los egresados universitarios, son temas recurrentes en las reflexiones sobre el papel de la universidad como formadora de individuos éticos. Tradicionalmente, en México el plagio ha sido considerado una actividad inmoral que debe combatirse, esencialmente, desde el plano legal. Esta mirada punitiva ha llevado a la mayoría de las universidades a proponer códigos de ética y endurecer las sanciones para los infractores sin que ello suponga, en la práctica, una disminución en los casos de conductas académicas deshonestas entre estudiantes y profesores.

Hoy sabemos que el plagio es un fenómeno de gran complejidad, que involucra factores de diversa índole que dificultan incluso su propia conceptualización; en el medio académico el plagio supone mucho más que copiar y atribuirse una obra ajena pues, como bien lo señala Girón (2015: 6-7), también se plagia cuando se toman ideas o palabras escritas por otros sin reconocer de forma directa haberlo hecho; cuando se copia lo dicho por otro en un discurso o dictado sin hacer referencia a la persona que lo dijo, o cuando se imita y reproduce un texto casi de forma idéntica.

Dada su estrecha relación con los procesos de producción escrita, actualmente, en el campo de los estudios del lenguaje existe un enorme interés por examinar el plagio como un fenómeno lingüístico, al tratarse esencialmente de un uso del lenguaje y no solo de la violación de reglas o principios éticos (Pecorari, 2010). Al estar directamente vinculado con los procesos de aprendizaje de las prácticas de literacidad propias de la formación académica y disciplinar, se reconoce que el plagio también debe ser estudiado como un fenómeno que se gesta en la dinámica de la producción del texto mismo. Lo anterior lleva a diferentes autores a proponer mecanismos de prevención del plagio que, sin hacer a un lado los códigos de ética, se centren en la enseñanza explícita a los estudiantes universitarios de los procesos de producción textual y, específicamente, de los recursos lingüístico-discursivos que los escritores expertos utilizan en la generación de textos originales en los distintos ámbitos del saber académico-científico (Bolívar, 2020; Borg, 2009, 2019). 
En este entendido, partimos del reconocimiento de que la escritura académica requiere de la adquisisción de habilidades específicas de manejo de información que solo se desarrollan en la medida en que se conocen las estrategias discursivas utilizadas por las diferentes comunidades disciplinares para generar conocimiento nuevo sobre la base de lo indagado y difundido por otros (Venegas et al., 2013; Pecori, Shaw, 2019; Castro, Sánchez, 2016). Sostenemos que, en la mayoría de los casos, el plagio que cometen los estudiantes en el posgrado es un acto originado por el desconocimiento de los recursos lingüísticos que permiten al escritor atribuir el conocimiento a las voces de la tradición. Se trata de intentos fallidos en la construcción del diálogo entre pares, característico de la comunicación especializada, y que se hace evidente en textos que presentan segmentos con copias literales de una o más fuentes, mezclados con secciones que han sido escritas por el estudiante de manera más o menos autónoma. Por lo mismo, en este trabajo nos centramos en lo que algunos autores denominan plagio textual (Pecorari, 2010), que es el que comete la mayoría de los estudiantes al escribir sus trabajos escolares cuando usan palabras o ideas tomadas de diversas fuentes sin atribuirlas a los autores originales, pero sin tener la abierta y clara intención de asumirlas como propias. Por lo tanto, nuestro objetivo es mostrar, a partir de un estudio exploratorio centrado en la identificación de marcas de atribución del conocimiento en borradores de tesis de maestría en el área de humanidades, las estrategias que los estudiantes utilizan para generar textos propios desde el conocimiento disciplinar contenido en otras fuentes. Nuestro interés es determinar el grado de conocimiento que los productores de las tesis tienen de las funciones retóricas y discursivas de los recursos de atribución, así como el grado de plagio textual presente en sus textos.

A fin de cumplir nuestro objetivo, en primer lugar, nos detenemos en el problema del plagio en el ámbito académico y su relación con los procesos de aprendizaje de la escritura disciplinar. En segundo lugar, nos centramos en la descripción de los recursos de atribución del conocimiento presentes en 11 borradores de tesis de grado de una maestría en el área de humanidades y su relación con el plagio textual. Finalmente, discutimos la pertinencia de replantear los mecanismos tradicionales de prevención del plagio en el posgrado, para incorporar una mirada lingüística que nos permita entender el fenómeno del plagio textual como parte de un proceso de aprendizaje que requiere de la orientación explícita a través de cursos de escritura académica impartidos a lo largo del currículum o, en su defecto, en cursos específicos de escritura disciplinar.

\section{Marco teórico}

\section{El plagio y el aprendizaje de la escritura académico-científica}

Las disciplinas que convergen en la universidad están constituidas por un conjunto de individuos que han sido formados bajo una lógica propia, derivada de su proceso de generación de conocimiento y del desarrollo de estrategias teóricas y metodológicas comunes. Por lo mismo, 
los miembros que conforman estas comunidades aprenden a dominar el conocimiento disciplinar y las herramientas para difundirlo, básicamente, a través de un proceso formal de escolarización que inicia en la licenciatura y se consolida en los estudios de posgrado. Dado que se trata de un ámbito de expertos, son precisamente los profesores de carrera y los investigadores adscritos a los distintos departamentos o áreas académicas los encargados de definir el currículo y determinar las competencias y los valores que debe desarrollar todo aquel que se forma en un determinado campo disciplinar; también son quienes regulan la incorporación de los nuevos miembros a las comunidades académicas y sancionan sus malas prácticas. Entre estas últimas, destacan todas las relacionadas con acciones académicas y profesionales deshonestas y, en particular, aquellas asociadas con el plagio.

Generalmente, en el medio universitario se concibe el plagio como un atentado contra la propiedad intelectual y, en consecuencia, una falta a la ética académica y profesional. Por lo mismo, en su definición y tratamiento se toman como base el fomento de una cultura de la integridad y honestidad académicas que, a través de los códigos de ética institucionales, conminan a los miembros de la comunidad a apegarse al rigor científico en la generación, difusión y divulgación del conocimiento. Prácticamente en todos estos códigos se insiste en el carácter fraudulento del plagio y se establece que la honestidad e integridad académicas se cultivan cuando se cita a las fuentes de ideas, textos, datos, imágenes, gráficos, etc., utilizados en los trabajos escolares y de investigación. Por lo mismo, se advierte a la comunidad de los riesgos e implicaciones académicas y legales que puede enfrentar aquel que manipule, invente o altere información, o copie fragmentos o textos completos sin indicar el origen de la información. Sin embargo, esta mirada estrictamente sancionadora no permite apreciar en su amplitud el carácter multifacético del plagio, y desestima las posibilidades de combatirlo dentro de las aulas, a través de planes de intervención orientados a la enseñanza de las prácticas disciplinares básicas, como los métodos de indagación, producción y difusión de saberes a través de la escritura académico-científica.

Hoy en día existe un amplio interés por acercarse al plagio desde perspectivas lingüísticas (Abasi et al., 2006; Pecorari, 2008; Li, Pearson, 2012; Pecorari, Shaw, 2019). Se parte del reconocimiento de que la copia literal o cuasi literal que cometen muchos de los estudiantes en sus trabajos escolares no está originada, en su mayoría, por un interés fraudulento, sino por la poca experiencia que han tenido con la escritura especializada y los recursos para incorporar conocimientos ajenos en sus propios textos. De ahí que algunos autores propongan el término plagio textual (Pecorari, 2008; Li, Pearson, 2012) para referirse específicamente al plagio que se comete en el entorno escolar y que consiste en usar palabras o ideas de otras fuentes sin atribuirlas apropiadamente. De este modo, la diferencia entre el plagio prototípico y el plagio textual estaría en su intención. El primero se caracteriza por ser a todas luces un embuste y el segundo, es en esencia un acto de desconocimiento e incluso de ingenuidad por parte del escritor, en la mayoría de los casos un aprendiz. 
En esta línea, no pocos autores interesados en la escritura académica se han dedicado a estudiar el plagio textual (Pecorari, Shaw, 2019; Davis, Morley, 2019). Por ejemplo, Pecorari (2010) señala que este tipo de plagio está frecuentemente asociado con la incorporación de secciones de textos extraídos de distintas fuentes y que los estudiantes mezclan con fragmentos escritos por ellos mismos. A esta mixtura o cruce de textos originales con secciones copiadas y pegadas sin indicar su origen, es lo que se conoce como patchwriting (Howard, 1999). El patchwriting o"escritura parchada" es un fenómeno sumamente común en los textos escolares; se trata de intentos de los estudiantes de apropiarse de los discursos especializados a través de la imitación y es prácticamente inevitable, pues es un mecanismo propio del aprendizaje del discurso disciplinar. Por lo mismo, al tratarse de un producto de aprendizaje de recursos lingüísticos y discursivos, su tratamiento no puede reducirse a advertencias y sanciones. Su abordaje requiere de un acercamiento didáctico que muestre a los estudiantes las relaciones que se establecen entre los textos y el papel que juegan los recursos lingüísticos de atribución del conocimiento (propio/ajeno) en la construcción de textos originales.

Queda claro, entonces, que el plagio textual está directamente relacionado con la incorporación de los estudiantes a una nueva cultura de lo escrito y, particularmente, con aquellos aspectos conectados con la configuración de una identidad como escritores académicos y miembros de una comunidad disciplinar.

\section{Atribución del conocimiento en las tesis de grado y su relación con el fenómeno del plagio textual}

Hemos señalado que el plagio textual que comete un número significativo de estudiantes puede explicarse como un intento fallido de afiliación que consiste en imitar los registros del discurso académico tomando prestadas las voces de otros, sin indicarlo explícitamente y sin una intención de engañar. Si bien este fenómeno es muy común en los trabajos escolares de estudiantes de licenciatura, también está presente en las producciones escritas de un número significativo de estudiantes de posgrado, particularmente en las tesis de grado.

La tesis es un género clave en la formación de los jóvenes investigadores. Prácticamente todas las maestrías y doctorados solicitan a sus estudiantes la elaboración de una investigación original que contribuya a ampliar los campos de conocimiento del área de formación. En ella, el escritor debe justificar la pertinencia de su aportación al campo de conocimiento, demostrar dominio suficiente del tema y de los métodos de investigación, además de manejar las convenciones de escritura disciplinar. Este proceso no es nada fácil pues los estudiantes deben tomar decisiones sobre la mejor manera de poner en escena, a través del lenguaje, su perspectiva sobre el fenómeno que pretenden comunicar, así como la pertinencia de la imagen que de sí mismos y de sus potenciales interlocutores construyen en ese intercambio (comunicación entre pares). De igual forma, determinan cuáles son los mecanismos más apropiados para incorporar 
su voz y establecer un diálogo con las voces de la tradición disciplinar, materializadas en la tesis mediante los recursos de cita y referencia. Por lo mismo, las exigencias de escritura en este nivel de formación obligan a los estudiantes a hacer más que simplemente resumir o sintetizar información proveniente de fuentes primarias. Deben asumir posturas, evaluar marcos conceptuales, construir opiniones fundamentadas y gestionar una imagen de escritor disciplinar, a partir de la construcción de una voz de autor que les permita expresar esas opiniones. Evidentemente, este proceso requiere del reconocimiento de las voces de la tradición expresada en las ideas y planteamientos de los autores referidos en los textos especializados, y de la comprensión de cómo esos conocimientos o saberes se construyen o reconstruyen con la múltiple intervención de las diversas voces que participan en el diálogo académico.

La noción de diálogo en este contexto de interacción es sumamente importante, pues significa que la escritura académica supone interactuar con los discursos que se proponen como marcos de referencia para la comprensión e interpretación de las diversas situaciones y conocimientos contenidos en los diversos documentos disciplinares. Sin embargo, a pesar de su importancia en la comunicación de saberes, este proceso de diálogo y su consecuente posicionamiento discursivo, no es una habilidad que los estudiantes desarrollan por el simple hecho de escribir trabajos escolares; para generar una discusión sobre un determinado aparato conceptual, el estudiante debe aprender a producir argumentos partiendo de la revisión de fuentes y la incorporación de información a través de los recursos de la cita y referencia (voz ajena), y expresarlos con su propia voz.

Dado que el análisis del fenómeno se ubica en el análisis del discurso académico, cabe aquí aclarar que cuando nos referimos a la voz, lo hacemos en los mismos términos que refieren Hyland y Sancho (2012) al abordar el discurso académico; es decir, a la posibilidad del escritor de manifestarse explícitamente en sus textos, de hacerse visible mediante el uso consciente y responsable de recursos lingüísticos que le permiten manifestar una postura frente a lo dicho por otros escritores, evaluar dicha información y expresar una propia opinión. Es importante señalar que la incorporación de la voz ajena a través de la cita y de la voz propia en primera persona (singular/plural), es parte de los recursos retóricos que utilizan los escritores para crearse una imagen de autoridad frente a sus lectores, que les permita defender posturas y expresar sus puntos de vista sobre lo planteado por otros autores (Castro, Sánchez, 2016). Es así que la atribución del conocimiento a los autores de la tradición disciplinar, mediante el recurso de la cita, se convierte en un aspecto relevante en la construcción de autoría, pues hace referencia a la responsabilidad del escritor de indicar el origen de los planteamientos traídos a su texto y el papel que dichos argumentos juegan en la construcción de la perspectiva propia (Hyland, 2002; Siddharthan, Teufel, 2007). Recordemos que en el medio académico, el análisis y validación de esos argumentos siempre se realiza a la luz de las aportaciones de otros autores (fuentes especializadas). 
Por lo mismo, no es de extrañar que la voz ajena, expresada en la cita textual, el parafraseo y la alusión a otros textos, sea un aspecto de la escritura académica estudiado desde perspectivas muy diversas. Así, por ejemplo, desde la lingüística enunciativa, la cita textual y el parafraseo han sido abordados como manifestaciones de la heterogeneidad, es decir, marcas que nos permiten introducir las voces de otros autores en un texto, revelando con ello las huellas de otros discursos (Massi, 2005). Se trata de lo que comúnmente se ha llamado "discurso referido" (Brunetti, 2009), y que no solo supone reproducir un enunciado a través de diferentes mecanismos tipográficos y gramaticales, sino también poner a distancia dicho enunciado para reconocerlo y/o deslindarse de él. Desde esta perspectiva, las voces de la tradición no hablan en el sentido material del término sino que lo hacen a través del escritor, quien expresa su punto de vista, posición o actitud frente a esos dichos. Así, la actitud del escritor frente a las voces ajenas puede ser de alejamiento o de adhesión y, para ello, el escritor separa su discurso de las palabras de otros por medio de marcas tipográficas como las comillas, o marcas gramaticales, en ocasiones muy sutiles y difíciles de distinguir, que se presentan a través del parafraseo. En este marco enunciativo, tradicionalmente se han identificado tres tipos de discurso referido o reproducido: el discurso directo, el discurso indirecto y el discurso indirecto libre (García, Tordesillas, 2001; Gallucci, 2016).

En contraste, para la perspectiva retórica (Perelman, Olbrechts-Tyteca, 1989), la cita no solo es la marca de otro enunciador en el discurso; aquí, además, es vista como un recurso de persuasión que el escritor puede elegir para poner en marcha una estrategia argumentativa apelando a la voz de autoridad. Aquí se aprovecha la credibilidad de la que goza una persona (autor) considerada "una autoridad en el tema" para darle más peso a las ideas que se quieren defender. A través de la cita, se negocia y establecen consensos con el interlocutor. Por lo mismo, en el contexto académico, la introducción de ideas o palabras ajenas no tiene una mera función descriptiva o informativa; quien escribe evalúa esas ideas, se adhiere a ellas o las rechaza, haciendo así avanzar la argumentación. Se entiende, entonces, que una de las funciones de la cita es la de apoyar la postura defendida en el texto, o de refrendar determinadas posiciones que son necesarias para sostener la argumentación; otra función es la de servir de medio para incorporar ideas que son potencialmente fuente de debate, por lo mismo, se recurre a la voz de autoridad como una garantía indirecta de su veracidad. Así, desde esta perspectiva se habla básicamente de dos tipos de cita: la de autoridad y la polémica (Navarro, 2012).

Finalmente, desde una perspectiva discursiva, la cita es vista como el principal mecanismo que tienen los escritores para construir conocimiento sobre la base de las ideas y los saberes de otros (Hyland, 2002). Desde esta línea se reconoce que el uso de un tipo particular de cita, junto con las convenciones formales relacionadas con los estilos de presentar las referencias, las notas y las tablas, por ejemplo, forman parte de prácticas discursivas más complejas en las que los autores de los textos expresan su relación con sus lectores, con su objeto de estudio y con el texto 
mismo que producen. Así, por ejemplo, Beke (2008) identifica cinco tipos de cita presentes en la comunicación científica y académica escrita en español: citas destacadas o no integradas, citas integradas, citas de parafraseo, citas de apoyo, citas de expansión.

Ahora bien, ¿cuál es la relación que se establece entre las fallas o errores en la atribución del conocimiento ajeno que comenten los estudiantes de posgrado al momento de escribir sus tesis con el plagio textual? Como ya lo señalamos, el plagio textual es un fenómeno inevitable en la formación de los jóvenes universitarios, sin embargo, es importante establecer lo que en un texto escolar o académico puede considerarse una imitación o parafraseo "aceptable" y lo que no.

Davis y Moley (2018) han señalado que la imitación verbal y, en consecuencia, la imitación de los registros escritos es parte del desarrollo natural del ser humano y de sus procesos de aprendizaje dentro de sus entornos culturales, incluido el contexto escolar. Por lo mismo, no es nada raro hallar prácticas de imitación del discurso ajeno en la escritura de los jóvenes universitarios al tratarse, como lo señalamos líneas arriba, de intentos de afiliación a las comunidades discursivas con las que están obligados a interactuar. Sin embargo, existen límites en esa imitación que no quedan del todo claros para los jóvenes escritores. Por ejemplo, Howard (1999) mostró que algunos estudiantes universitarios elaboran sus escritos parafraseando de manera muy elaborada fuentes primarias; están convencidos de que al cambiar palabras, estructuras gramaticales o utilizar sinónimos, están generando textos auténticos, por lo que no es necesario indicar la fuente.

Con lo anterior, queda claro que en este proceso de aprendizaje es indispensable guiar al estudiante en lo que está permitido imitar y lo que necesariamente debe atribuir a las fuentes consultadas. Por lo tanto, en este trabajo hacemos la distinción de lo que podemos llamar imitación legítima y copia ilegítima. La imitación legítima supone, precisamente, la reproducción de convenciones establecidas por las comunidades disciplinares, y comprende la imitación de formas de organizar la información dentro de los textos, por ejemplo, la estructura retórica de los géneros de la comunicación académica como el ensayo, la tesis o el artículo de investigación; también incluye la posibilidad de retomar o reusar frases académicas ("la reciente evidencia sugiere"; "de acuerdo con lo hallado en este estudio", "los resultados de la investigación muestran", "a partir de lo hallado podemos concluir"; "iniciamos nuestra reflexión"; "el presente trabajo tiene como objetivo", etc.). Por su parte, la copia ilegítima puede ser el resultado de un acto claro de engaño, por ejemplo, tomar fragmentos, datos, tablas de diversas fuentes y mezclarlos con párrafos propios usando el recurso de copiar y pegar; o parafrasear segmentos de textos sin indicar la fuente, o citar a terceros sin haber consultado nunca la fuente original. Pero también puede deberse, como lo hemos indicado a lo largo de esta exposición, a fallas en la atribución del conocimiento, por lo que es importante ahondar en esta problemática que, por lo menos en nuestro país, no ha sido del todo estudiada. 


\section{Metodología}

El presente estudio posee un enfoque cualitativo de corte exploratorio enmarcado en el Análisis del Discurso, y tiene como objetivo describir la manera como los estudiantes de una maestría en humanidades utilizan los recursos de atribución del conocimiento en los borradores de sus tesis de grado. Nuestra intención es identificar el uso que estos estudiantes hacen de los recursos de cita y referencia, a fin de determinar su relación con el plagio textual.

\section{Muestra}

La muestra está conformada por borradores de la introducción y un capítulo teórico de 11 tesis en proceso de producción, en una maestría en el área de humanidades de una universidad pública mexicana. Si bien se trata de una pequeña muestra que, por lo mismo, no nos permite llegar a resultados concluyentes, su análisis nos da pauta para futuros estudios que nos ayuden a profundizar en la investigación sobre la construcción de autoría, la atribución del conocimiento y el plagio textual en la formación de jóvenes investigadores.

\section{Procedimiento}

El análisis de los textos que conforman la muestra se llevó a cabo en tres fases previamente establecidas. La primera fase consistió en la identificación y segmentación de la estructura retórica de las introducciones y los capítulos teóricos a examinar. Para ello, nos remitimos a los pasos o etapas del evento comunicativo en este contexto específico de producción (posgrado), y a los propósitos que este evento y sus etapas cumplen; por lo mismo, retomamos la noción de movimiento, que desde los estudios de las lenguas con propósitos específicos se ha propuesto para identificar las unidades retóricas que conforman la estructura de un texto, en este caso la introducción y el capítulo teórico del género tesis de grado, para establecer la organización prototípica de nuestra muestra (Swales, 2004; Parodi, 2008). Esta primera fase del análisis nos permitió identificar la estructura básica, no rígida y válida en el área de las humanidades, para las secciones analizada. Una vez analizada la estructura de las introducciones y los capítulos, la segunda fase consistió en la identificación manual de las secciones de la muestra en la que los autores señalan explícitamente las voces ajenas a través de los recursos de cita para, posteriormente, identificar tipo y función de los mismos. Finalmente, la tercera fase del análisis consistió en la utilización del programa Turnitin para identificar las secciones de los textos que presentan coincidencias o similitudes con textos de otros autores. Esta última fase del análisis nos sirvió para identificar la presencia o ausencia de plagio textual en los avances de tesis de los estudiantes. 


\section{Resultados y discusión}

Estructura retórica

El concepto de género discursivo es central para el análisis de los textos producidos por comunidades específicas en contextos determinados como el que aquí nos ocupa. La noción, retomada de Bajtín (1985), hace referencia a las formas relativamente estables que adquieren los discursos en los distintos ámbitos de comunicación en el contexto de la vida social. Por lo tanto, al ser patrones estables, los géneros representan las formas como las distintas comunidades que organizan sus experiencias y las comparten con los demás miembros de la comunidad. En el caso de la vida académica, muchos de sus géneros son "cerrados"; es decir, géneros que se producen y circulan exclusivamente en el interior de la comunidad (Maingueneau, 2002). Por lo mismo, presentan patrones de organización de la información asociados a objetivos comunicativos bastante estables, lo que aquí llamamos estructura retórica (Swales, 2004; Parodi, 2008).

Dado que su propósito es comunicar los resultados de una indagación sustentada en métodos científicos y razonamientos teóricos, la tesis de grado guarda enorme semejanza con el artículo de investigación científica. Venegas et al. (2016), por ejemplo, hablan de una estructura tradicional simple en las tesis de licenciatura, compuesta por cinco movimientos: introducción, revisión de la literatura o marco teórico, métodos, resultados, discusión y conclusiones; estructura similar a la descrita por otros autores al analizar tesis de distintas disciplinas y grados académicos (Paltridge, Starfield, 2007; Tapia, Burdiles, 2012; Morales et al., 2020).

Así, la estructura retórica de la introducción y del marco teórico halladas en nuestra muestra presentan un patrón homogéneo; hecho que muy probablemente se debe a la orientación metodológica recibida por los estudiantes en el Seminario de Investigación I, en el que trabajaron estas dos secciones de la tesis, y en el que se aplicaron los acuerdos establecidos por el consejo académico del posgrado en cuanto a las características que la tesis debe presentar en esta maestría en particular. En lo que respecta a la Introducción, en los 11 casos revisados, esta precede al capítulo teórico en el que se hace la revisión de la literatura y se establece el marco conceptual que guía el análisis y reflexión sobre la problemática abordada. La introducción presenta un patrón conformado por seis movimientos: 1) presentación del problema; 2) breve estado del conocimiento; 3) justificación; 4) objetivo; 5) metodología y 6) organización del trabajo. En la tabla 1 presentamos lo hallado al analizar la muestra. Para facilitar el análisis, hemos identificado a cada avance de tesis con la etiqueta "texto" agregando un dígito identificador. La presencia del movimiento está señalada con el signo (-). 
Tabla. 1. Estructura retórica de la Introducción

\begin{tabular}{|l|c|c|c|c|c|c|c|c|c|c|c|}
\hline Movimientos & $\begin{array}{c}\text { Texto } \\
\mathbf{1}\end{array}$ & $\begin{array}{c}\text { Texto } \\
\mathbf{2}\end{array}$ & $\begin{array}{c}\text { Texto } \\
\mathbf{3}\end{array}$ & $\begin{array}{c}\text { Texto } \\
\mathbf{4}\end{array}$ & $\begin{array}{c}\text { Texto } \\
\mathbf{5}\end{array}$ & $\begin{array}{c}\text { Texto } \\
\mathbf{6}\end{array}$ & $\begin{array}{c}\text { Texto } \\
\mathbf{7}\end{array}$ & $\begin{array}{c}\text { Texto } \\
\mathbf{8}\end{array}$ & $\begin{array}{c}\text { Texto } \\
\mathbf{9}\end{array}$ & $\begin{array}{c}\text { Texto } \\
\mathbf{1 0}\end{array}$ & $\begin{array}{c}\text { Texto } \\
\mathbf{1 1}\end{array}$ \\
\hline $\begin{array}{l}\text { Presentación } \\
\text { del problema }\end{array}$ & - & - & - & - & - & - & - & - & - & - & - \\
\hline $\begin{array}{l}\text { Estado del co- } \\
\text { nocimiento }\end{array}$ & - & - & - & - & & - & - & - & & - & - \\
\hline Justificación & - & - & - & - & & - & - & - & - & - & - \\
\hline Objetivo & - & - & - & - & - & - & - & - & - & - & - \\
\hline Metodología & & & - & & - & & & & & - & - \\
\hline $\begin{array}{l}\text { Organización } \\
\text { del trabajo }\end{array}$ & - & & & & & - & - & & & & \\
\hline
\end{tabular}

Fuente: Elaboración propia.

Como se observa, existe en esta sección una pequeña variabilidad en los movimientos identificados que se puede explicar, en parte, porque se trata de borradores que se encuentran en constante corrección. Una comparación de estos textos con las versiones finales resultará interesante para rastrear el proceso de construcción de esta sección específica e identificar el patrón que siguen los estudiantes al realizar el planteamiento del problema y presentar la investigación.

En cuanto al capítulo teórico, este se concentra en la revisión crítica de estudios previos y en el establecimiento del marco conceptual que guía el trabajo de investigación. Esto coincide con lo hallado por otros autores al analizar los capítulos de las tesis de licenciatura y posgrado (Morales et al., 2020). A diferencia de lo que ocurre con la introducción, este capítulo no presenta un patrón homogéneo. Se trata de una macrosección dividida en secciones menores identificadas con subtítulos que varían en número y extensión (véase la tabla 2).

Tabla 2. Estructura retórica del capítulo teórico

\begin{tabular}{|l|c|c|c|c|c|c|c|c|c|c|c|}
\hline Movimientos & $\begin{array}{c}\text { Texto } \\
\mathbf{1}\end{array}$ & $\begin{array}{c}\text { Texto } \\
\mathbf{2}\end{array}$ & $\begin{array}{c}\text { Texto } \\
\mathbf{3}\end{array}$ & $\begin{array}{c}\text { Texto } \\
\mathbf{4}\end{array}$ & $\begin{array}{c}\text { Texto } \\
\mathbf{5}\end{array}$ & $\begin{array}{c}\text { Texto } \\
\mathbf{6}\end{array}$ & $\begin{array}{c}\text { Texto } \\
\mathbf{7}\end{array}$ & $\begin{array}{c}\text { Texto } \\
\mathbf{8}\end{array}$ & $\begin{array}{c}\text { Texto } \\
\mathbf{9}\end{array}$ & $\begin{array}{c}\text { Texto } \\
\mathbf{1 0}\end{array}$ & $\begin{array}{c}\text { Texto } \\
\mathbf{1 1}\end{array}$ \\
\hline $\begin{array}{l}\text { Introducción o } \\
\text { presentación } \\
\text { del capítulo }\end{array}$ & - & - & & & - & - & & & - & \\
\hline $\begin{array}{l}\text { Secciones } \\
\text { subtítuladas }\end{array}$ & 6 & 6 & 7 & 3 & 6 & 3 & 4 & 5 & 4 & 5 & 6 \\
\hline $\begin{array}{l}\text { Cierre } \\
\text { Referencias } \\
\text { bibliográficas }\end{array}$ & - & - & - & - & - & - & - & - & - & - & - \\
\hline
\end{tabular}

Fuente: Elaboración propia. 
Como se aprecia, la estructura del capítulo dedicado al desarrollo del marco conceptual no tiene un patrón establecido. Tres de los 11 capítulos revisados presentan un primer párrafo que sirve para presentar el objetivo del capítulo, su contenido y la manera como se desarrolla; también contienen un párrafo de cierre del capítulo que resume lo tratado por el autor y señala su conexión con el capítulo siguiente. El promedio de palabras por capítulo es de nueve mil, lo que equivale aproximadamente a veinticinco cuartillas, incluidas las referencias bibliográficas.

\section{Mecanismos de atribución del conocimiento}

Tanto las introducciones como los capítulos teóricos analizados presentan abundantes marcas de atribución del conocimiento a autores de la tradición disciplinar. Lo anterior se explica debido a que en estas secciones el autor comunica a la comunidad su aportación al campo de formación y, para ello, debe apoyarse en una revisión general de la literatura que conforma el estado del conocimiento relacionado con la problemática abordada. Es a partir del diálogo que logra construir con las voces expertas, que el autor genera la línea argumentativa que fundamenta la pertinencia y novedad de su aportación. Particularmente, en la tesis de posgrado, los capítulos que conforman el marco de referencia son, en esencia, una discusión pormenorizada en la que el estudiante debe mostrar su capacidad de organizar información, describir, evaluar y conceptualizar o reconceptualizar temáticas para dar sentido a su propio discurso. Las tablas 3,4 y 5 resumen la cantidad, tipo y función de los recursos de atribución hallados en las introducciones y capítulos que conforman la muestra.

Tabla 3. Número de citas por sección

\begin{tabular}{|c|c|c|c|c|}
\hline \multirow{2}{*}{ Muestra } & Introducción & Capítulo teórico & \multirow{2}{*}{ Total de citas } & $\begin{array}{c}\text { Total de obras } \\
\text { referidas en la } \\
\text { bibliografía }\end{array}$ \\
\cline { 2 - 3 } & No. de citas & No. de citas & 89 & 31 \\
\hline Texto 1 & 17 & 62 & 76 & 42 \\
\hline Texto 2 & 12 & 64 & 39 & 29 \\
\hline Texto 3 & 0 & 39 & 116 & 38 \\
\hline Texto 4 & 37 & 79 & 66 & 70 \\
\hline Texto 5 & 0 & 66 & 50 & 33 \\
\hline Texto 6 & 6 & 44 & 51 & 28 \\
\hline Texto 7 & 14 & 37 & 63 & 37 \\
\hline Texto 8 & 9 & 54 & 84 & 46 \\
\hline Texto 9 & 16 & 58 & 54 & 34 \\
\hline Texto 10 & 7 & 49 & 82 & 42 \\
\hline Texto 11 & 21 & 61 & & \\
\hline
\end{tabular}

Fuente: Elaboración propia. 
La presencia de citas es muy variable y, como es de esperar, la mayor cantidad se encuentra en la sección que desarrolla el aparato teórico que sirve de base a la argumentación y al posicionamiento de los autores. Las formas que utilizan los estudiantes para presentar información ajena es similar a la reportada por otras investigaciones sobre la tesis de grado (Meza, 2015; Venegas et al., 2013). Por un lado, la cita incorpora la voz ajena a través de la cita directa (cita textual) o indirecta (parafraseo); por otro, a través de la mención refiere a autores, investigaciones, proyectos u obras.

Tabla 4. Usos de la cita identificados en la Introducción

\begin{tabular}{|c|c|c|c|c|}
\hline & \multicolumn{2}{|c|}{$\begin{array}{c}\text { Para introducir voces de } \\
\text { otros autores }\end{array}$} & $\begin{array}{c}\text { Para referir autores } \\
\text { e investigaciones }\end{array}$ & Total de citas \\
\cline { 2 - 3 } Muestra & Cita directa & Parafraseo & 8 & 17 \\
\hline Texto 1 & 0 & 9 & 3 & 12 \\
\hline Texto 2 & 5 & 4 & 0 & 0 \\
\hline Texto 3 & 0 & 0 & 9 & 37 \\
\hline Texto 4 & 8 & 20 & 0 & 0 \\
\hline Texto 5 & 0 & 0 & 1 & 6 \\
\hline Texto 6 & 5 & 0 & 6 & 14 \\
\hline Texto 7 & 3 & 5 & 0 & 9 \\
\hline Texto 8 & 4 & 5 & 3 & 16 \\
\hline Texto 9 & 9 & 4 & 0 & 7 \\
\hline Texto 10 & 5 & 2 & 6 & 21 \\
\hline Texto 11 & 8 & 7 & & 0 \\
\hline
\end{tabular}

Fuente: Elaboración propia.

Generalmente, la introducción en la tesis de grado se construye a partir de la voz propia, lo que explica la escasa presencia en nuestra muestra de recursos de citación, en comparación con los capítulos teóricos. Esto es así porque en la introducción el autor debe posicionarse frente al hecho estudiado para indicar los vacíos en el ámbito de conocimiento, problematizar, enmarcar la investigación en una tradición, justificar su pertinencia e indicar sus propósitos. La presencia de citas en esta sección, aunque reducida, es importante, pues para lograr el posicionamiento, el autor debe tomar como punto de referencia las aportaciones realizadas por los expertos al introducir conceptos, teorías o presentar el desarrollo del ámbito de conocimiento en el que se ubica el problema abordado. Lo importante aquí es la función que los estudiantes dan al recurso de citación en esta sección específica de sus tesis ya que, como podemos observar en la tabla anterior, en la mayoría de los casos la cita funciona como recurso para la introducción de voces 
que sirvan de apoyo para la construcción del apartado expositivo-argumentativo; es decir, se retoma la voz del experto como voz de autoridad a la que se adhieren para fundamentar sus opiniones sin polemizar. Un dato interesante es la escasa mención de autores o investigaciones en la construcción del nicho de oportunidad de la investigación, lo que muestra escasa referencia a estudios previos y revela una mínima indagación de fuentes por parte de los estudiantes. De igual forma, llama la atención el caso del texto 3 y el texto 5; en estas introducciones, los autores construyen el espacio de indagación sin remitir a ninguna fuente, lo que también sugiere problemas en el establecimiento del territorio de estudio y adjudicación de discursos ajenos.

En cuanto al capítulo teórico, notamos un equilibrio entre la presencia de citas de incorporación de voces y aquellas utilizadas para mencionar autores o documentos (véase la tabla 4.) En la mayoría de los capítulos se hace un uso significativo de citas textuales que remiten a nociones, conceptos e ideas de otras fuentes y que permiten a los estudiantes construir los marcos teóricos que sustentan las tesis. Así, para la construcción del capítulo, los estudiantes realizan una selección de obras y autores que consideran relevantes en el contexto de la problemática abordada.

Tabla 5. Usos de la cita identificadas en el capítulo teórico

\begin{tabular}{|c|c|c|c|c|}
\hline \multirow[t]{2}{*}{ Muestra } & \multicolumn{2}{|c|}{$\begin{array}{c}\text { Para introducir } \\
\text { voces de otros } \\
\text { autores }\end{array}$} & \multirow{2}{*}{$\begin{array}{c}\text { Para referir autores } \\
\text { e investigaciones }\end{array}$} & \multirow[t]{2}{*}{ Total de citas } \\
\hline & $\begin{array}{c}\text { Cita } \\
\text { directa }\end{array}$ & Parafraseo & & \\
\hline Texto 1 & 19 & 40 & 3 & 62 \\
\hline Texto 2 & 37 & 27 & 0 & 64 \\
\hline Texto 3 & 21 & 18 & 0 & 39 \\
\hline Texto 4 & 31 & 40 & 8 & 79 \\
\hline Texto 5 & 28 & 34 & 4 & 66 \\
\hline Texto 6 & 11 & 16 & 2 & 29 \\
\hline Texto 7 & 14 & 20 & 6 & 40 \\
\hline Texto 8 & 31 & 29 & 4 & 64 \\
\hline Texto 9 & 9 & 26 & 1 & 36 \\
\hline Texto 10 & 5 & 39 & 5 & 49 \\
\hline Texto 11 & 45 & 18 & 0 & 61 \\
\hline
\end{tabular}

Fuente: Elaboración propia.

A diferencia de la introducción, en esta sección los recursos de citación, como procedimientos discursivos, tienen funciones muy específicas. Así, podemos observar que, tal como señala 
Beke (2008), la cita textual funciona en muchos casos como recurso de autoridad para indicar adhesión a los planteamientos del experto y mostrar que la exposición realizada se ajusta a las teorías referidas. En cuanto a la cita indirecta, esta es utilizada por los estudiantes como apoyo para la construcción de la línea argumentativa al defender ideas y expresar opiniones. También es común el parafraseo con fines de demostración de conocimiento en el tema.

En resumen, esta primera exploración de los mecanismos de cita presentes en la muestra, revela que la elección del tipo, la función y el número de citas dependen de los propósitos retóricos de cada escritor, pero también puede deberse a las experiencias previas de escritura que los autores han tenido a lo largo de su formación. Un estudio sobre las concepciones que los jóvenes escritores tienen con respecto a estos recursos puede ayudarnos a aclarar muchas de las interrogantes que surgen al observar los mecanismos de atribución de conocimiento propio y ajeno en la escritura inexperta.

\section{El plagio textual}

En lo que respecta al plagio textual, los reportes de similitud arrojados por la herramienta Turnitin evidencian un fenómeno que debe abordarse con cautela. Cada vez es mayor el número de expertos que señalan que el uso indiscriminado del software puede traducirse en prácticas contraproducentes por parte de profesores e instituciones educativas en su afán de combatir el plagio (Canzoneta, 2019, Sutherland-Smith, 2019). En este entendido, concordamos con estos autores cuando señalan que la herramienta no es un instrumento punitivo, sino un recurso de apoyo en el proceso de la enseñanza de los métodos, búsqueda de información y de escritura académica. De ahí que sea necesario establecer consensos, según criterios objetivos, que permitan decidir qué porcentaje de coincidencias pueden ser consideradas plagio, pues existen instituciones de educación superior que establecen $45 \%$ de coincidencias como aceptable, otras más se van al extremo de señalar que solo $15 \%$.

En este trabajo, no es de nuestro interés discutir el tema de los porcentajes de coincidencia para señalar a presuntos plagiarios, sino describir y explicar la presencia de similitudes entre los textos analizados y las fuentes referidas. Por lo mismo, nos centramos en el rastreo de coincidencias sin hacer evaluaciones de orden ético. Bajo este supuesto, en el proceso de identificación tomamos en cuenta todas las bases de datos del programa; y para centrar la atención en las secciones de textos que los estudiantes presentan como propias u originales, se excluyeron del resumen de coincidencias la bibliografía, las citas textuales, el parafraseo con referencia y las coincidencias menores a diez palabras que pueden ser considerados una copia legítima, como lo explicamos en nuestro marco referencial. En todos los textos que conforman la muestra se observan coincidencias en diferentes grados (véase la tabla 6). 
Tabla 6. Porcentaje de coincidencias

\begin{tabular}{|c|c|c|c|c|c|}
\hline Muestra & $\begin{array}{c}\text { Similitud } \\
\text { general }\end{array}$ & Palabras & Caracteres & $\begin{array}{c}\text { Mayor similitud } \\
\text { con una fuente }\end{array}$ & $\begin{array}{c}\text { Coincidencias } \\
\text { detectadas }\end{array}$ \\
\hline Texto 6 & $61 \%$ & 7,735 & 43,361 & $16 \%$ & 4,718 \\
\hline Texto 9 & $34 \%$ & 7,119 & 39,253 & $6 \%$ & 2,420 \\
\hline Texto 8 & $30 \%$ & 11,435 & 61,770 & $16 \%$ & 3,431 \\
\hline Texto 1 & $14 \%$ & 7,909 & 44,398 & $3 \%$ & 1,107 \\
\hline Texto 5 & $5 \%$ & 9,155 & 51,388 & $1 \%$ & 458 \\
\hline Texto 2 & $5 \%$ & 9,893 & 53,360 & $1 \%$ & 495 \\
\hline Texto 11 & $5 \%$ & 10,755 & 59,868 & $1 \%$ & 538 \\
\hline Texto 3 & $5 \%$ & 11,926 & 66,644 & $1 \%$ & 596 \\
\hline Texto 7 & $5 \%$ & 13,571 & 76,922 & $1 \%$ & 679 \\
\hline Texto 10 & $4 \%$ & 5,205 & 29,921 & $2 \%$ & 208 \\
\hline Texto 4 & $3 \%$ & 12,208 & 68,007 & $1 \%$ & 366 \\
\hline Promedio & $16 \%$ & 9,719 & 54,081 & $4 \%$ & 1,415 \\
\hline $\begin{array}{c}\text { Desviación } \\
\text { estándar }\end{array}$ & $19 \%$ & 2,542 & 14,093 & $6 \%$ & 1,537 \\
\hline Mediana & $5 \%$ & 9,893 & 53,360 & $1 \%$ & 637 \\
\hline
\end{tabular}

Fuente: Elaboración propia a partir de las coincidencias arrojadas por Turnitin.

Debido a que nuestro interés está orientado a identificar el plagio textual que se presenta frecuentemente mediante el patchwriting (Howard, 1999), pusimos especial atención en aqueIlas secciones marcadas por el software como coincidencias mayores. El análisis muestra, en todos los textos, una presencia de secciones cortadas y pegadas por los estudiantes, pero en una cantidad mucho menor a lo reportado por estudios realizados en tesis pregrado (Torres et al., 2018). Lo anterior puede explicarse, quizá, por el hecho de que en el posgrado, el estudiante se ve obligado a ir construyendo una imagen de autor que dé confianza a los lectores sobre la originalidad y validez de los argumentos y hallazgos. Creemos que mucha de esta copia literal está asociada con intentos malogrados de parafraseo sin referencia a las fuentes, lo que en este trabajo hemos denominado plagio ingenuo. Evidentemente, este es un punto que debe explorarse en investigaciones futuras.

Otro hallazgo significativo, asociado con la presencia de patchwriting, es que este se presenta en mayor cantidad en secciones en las que el estudiante debe argumentar a favor de planteamientos o ideas propias o ajenas. Lo que nos da pistas sobre la importancia de trabajar en los cursos de escritura académica o en los seminarios de tesis con el tema de la construcción de autoría pues, al parecer, en un intento de mostrar dominio de la temática abordada en la tesis, los estudiantes caen en la trampa de crearse una falsa imagen de autoridad retomando lo aportado por otras voces. Por lo mismo, creemos pertinente trabajar de manera puntual con 
las tesis la construcción del aparato argumentativo mostrándole los recursos que le permitirán situarse en relación con el conocimiento ajeno sin plagiar, y encontrar maneras satisfactorias de expresar sus propios argumentos. Hacer explícitas a los estudiantes las convenciones en el uso de la primera persona (singular/plural), y los recursos de posicionamiento discursivo en la comunicación académica, puede ayudarlos a construirse una imagen de escritor responsable que reconoce las aportaciones de otros, las analiza, comparte o refuta.

Creemos que al estar directamente relacionado con los procesos de afiliación por los que todo joven universitario debe transitar, este tipo de plagio puede evitarse, en buena medida, con programas de inmersión a las prácticas y códigos de la comunicación académica y científica, a través de programas de alfabetización académica y disciplinar, y cursos específicos de escritura especializada en licenciatura y posgrado.

\section{Conclusiones}

La construcción de un texto académico siempre es el resultado de una red de conexiones intertextuales que permite al escritor establecer un diálogo con sus pares. De ahí que el aprendizaje de los recursos de atribución del conocimiento sea fundamental en el proceso de formación de autores responsables y éticos.

La enseñanza explícita de estos recursos abona claramente al proceso de construcción identitaria como autores expertos, por el que todo estudiante de posgrado debe atravesar. En este proceso, el estudiante se ve en la necesidad de reportar lo que han dicho otras voces, pues a través de las citas y referencias refuerza sus argumentos, a la vez que se posiciona como conocedor del tema y demuestra que sus ideas se apoyan en planteamientos consensuados. La presencia de plagio textual en los borradores de tesis de grado revela la necesidad de trazar un plan de intervención que permita a los futuros investigadores aprender a escribir textos académico-científicos cumpliendo con las convenciones y principios de honestidad propios del quehacer científico. Esto es posible si se guía al estudiante en el proceso de examinación de los textos académicos y especializados propios de su área disciplinar y de los recursos discursivos que los caracterizan.

Abordar la enseñanza del discurso referido y la expresión de la voz propia, no es un tema de simples asuntos conceptuales; no basta decirle al estudiante qué es la cita y cómo se clasifica, o qué pronombres, deícticos y demás recursos léxicos debe utilizar para dejar constancia de su voz en el texto, o de las voces expertas con las que dialoga. Se trata de mostrarle la naturaleza de ese diálogo para que aprenda a ponerse en relación con los mundos referidos por la voz de tradición disciplinar, y así tomar las mejores decisiones sobre qué autor citar, con qué tipo de cita, en qué sección del texto y para qué hacerlo.

Finalmente, creemos que el acercamiento a las diversas formas de incorporación de la voz ajena y la construcción de la voz autoral plantea cuestiones complejas que se resolverán en la 
medida que se avance en el estudio y descripción de los géneros discursivos propios de la comunicación especializada en nuestra lengua. De igual forma, insistimos en que, al ser la universidad per se la institución promotora de estrategias para la generación y comunicación de conocimientos, es también la principal responsable de velar por una formación de calidad, ética e inclusiva, que promueva habilidades de lectura y escritura no solo con el objetivo de garantizar el éxito académico de sus estudiantes, sino también para lograr la real incorporación al mundo de la investigación científica de muchos de ellos.

\section{Referencias}

Abasi, A.; N. Akbari; B. Graves (2006). Discourse Appropriation, Construction of Identities and the Complex Issue of Plagiarism: ESL Students Writing in Graduate School. Journal of Second Language Writing, 15(2), 102-117. https://doi.org/10.1016/j.jslw.2006.05.001

Bajtín, M. (1985). Estética de la creación verbal (T. Bubnova, trad.). México: Siglo XXI.

Beke, R. (2008). El discurso académico: La atribución del conocimiento en la investigación educativa. Núcleo, 20(25), 13-36.

http://ve.scielo.org/scielo.php?script=sci arttext\&pid=S079897842008000100002\&lng=es\&tlng=en

Bolívar, A. (2020). El plagio y la construcción del conocimiento propio y ajeno en textos académicos. En M. Sánchez Camargo (coord.). Hacia una universidad libre de plagio. Mecanismos para su prevención, México: UDLAP, 83-113.

Borg, E. (2009). Local Plagiarism. Assessment and Evaluation in Higher Education, 34(4), 415-426. http://dx.doi.org/10.1080/02602930802075115

Borg, E. (2019). How does Intertextuality Inform Plagiarism? En Pecorari, D.; P. Shaw (eds.). Student Plagiarism in Higher Education. Nueva York: Routledge, 89-104.

Brunetti, P. (2009). El discurso referido. Formas canónicas y no canónicas de citación en la prensa diaria. Aspectos teóricos y didácticos. Buenos Aires: Comunic-Arte.

Castro, M. C.; M. Sánchez (2016). La formación de investigadores en el área de humanidades: Los retos de la construcción de la voz autoral en la escritura de la tesis de doctorado. Revista Signos, 49(Supl. 1), 30-51. https://dx.doi.org/10.4067/S0718-09342016000400003

Canzonetta, J. (2019). Can We Use Plagiarism Detection Services Responsibly? En Pecorari, D.; P. Shaw (eds.). Student Plagiarism in Higher Education. Nueva York: Routledge, 89-104.

Davis, D.; Morley, J. (2019). How Much Can You Copy? En Pecorari, D.; P. Shaw (eds.). Student Plagiarism in Higher Education. Nueva York: Routledge, 28-46.

Gaducci, M. J. (2016). El discurso referido en los manuales sobre análisis del discurso y pragmática lingüística. Lengua y Habla, (20), 200-224. https://www.redalyc.org/articulo.oa?id=511954843011

García, M. M.; M. Tordesillas (2001). La enunciación en la lengua. De la deixis a la polifonía. Madrid: Gredos. 
Girón, S. (2015). Anotaciones sobre el plagio. Bogotá: Universidad Sergio Arboleda.

https://www.usergioarboleda.edu.co/wp-content/uploads/2015/05/Libro-No-Plagio -Digital-1.pdf?db852a

Li, Y; Ch. Pearson (2012). Two First-year Student's Strategies for Writing from Sources: Patchwriting or Plagiarism? Journal of Second Language Writing 21(2), 165-180. https://doi.org/10.1016/j.jslw.2012.03.002

Howard, R. M. (1999). Standing in the Shadow of Giants: Plagiarists, Authors, Collaborators, Stamford: Ablex.

Hyland, K. (2002). Autority and Invisibility: Authorial Identity in Academic Writing. Journal of Pragmatics, (34), 1091-1112. https://doi.org/10.1016/S0378-2166(02)00035-8

Hyland, K.; C. Sancho (eds.) (2012). Stance and Voice in Written Academic Genres. Nueva York: Palgrave Macmillan.

Maingueneau, D. (2002). Analysis of an Academic Genre. Discourse Studies, 3(4), 319-342. https:// doi.org/10.1177/14614456020040030401

Massi, M. (2005). La cita en la comunicación académica escrita. Revista Iberoamericana de Educación, 36(5), 1-8. http://www.amsafelacapital.org.ar/images/201804/encuentros de escritura/massi m p 2005 01.pdf

Meza, P. (2015). La comunicación del conocimiento en el género Tesis de Lingüística: Comparación entre grados académicos. En Parodi, G.; G. Burdiles (eds.). Leer y escribir en contextos académicos y profesionales, Santiago: Planeta, 67-112.

Morales, A.; B. Perdomo; D. Cassany; E. Izarra (2020). Estructura retórica de tesis y trabajos de grado en odontología: Un estudio en Hispanoamérica y España. Íkala. Revista de Lenguaje y Cultura, 25(2), 373-393. https://revistas.udea.edu.co/index.php/ikala/article/view/337535/20802471

Navarro, F. (2012). La cita bibliográfica. En Natale, L. (coord.). En carrera: escritura y lectura de textos académicos y profesionales, Cole. Textos Básicos. Los Polvorines: Universidad Nacional de General Sarmiento, 179-192.

Paltridge, B.; S. Starfield (2007). Thesis and Dissertation Writing in a Second Language: a Handbook for Supervisors. Londres: Routledge.

Parodi, G. (ed.). (2008). Géneros académicos y géneros profesionales: Accesos discursivos para saber y hacer. Chile: Ediciones Universitarias de Valparaíso, Pontificia Universidad Católica de Chile.

Pecorari, D. (2010). Academic Writing and Plagiarism. A Linguistic Analysis. Londres: Continuum.

Pecorari, D.; S. Philip (eds.). (2019). Student Plagiarism in Higher Education. Nueva York: Routledge.

Perelman, CH.; L. Olbrechts-Tyteca (1989). Tratado de la argumentación. La nueva retórica. Madrid: Gredos.

Real Academia Española (2014). Diccionario de la lengua española. (23. ${ }^{a}$ ed.). España. https:// dle.rae.es

Savio, A. (2010). Las huellas del autor en el discurso académico: un estudio sobre tesis de psicoa- 
nalistas argentinos. Lenguaje, 38(2), 563-590. https://core.ac.uk/reader/11863340

Sutherland-Smith, W. (2019). Is Student Plagiarism still Serious Problem in Universities Today? En Pecorari, D.; P. Shaw (eds.). Student Plagiarism in Higher Education. Nueva York: Routledge, 47-61.

Swales, J. (1990). Genre Analysis: English in Academic and Research Settings. Cambridge: Cambridge University Press.

Tapia, M.; G. Burdiles (2012). La organización retórica del marco referencial en tesis de trabajo social. Alpha (Osorno), (35), 169-184. https://dx.doi.org/10.4067/S0718-22012012000200011

Torres, J.; J. Duart; M. Hinojosa (2018). Plagio, uso de Internet y éxito académico en la Universidad. JournalNewApproaches in Educational Research 7(2), 98-104.https://doi.org/10.7821/naer.2018.7.324

Venegas, R.; P. Meza; J. Martínez (2013). Procedimientos discursivos en la atribución del conocimiento en tesis de lingüística y filosofía en dos niveles académicos. RLA. Revista de Lingüística Teórica y Aplicada, 51(1), 153 179. https://dx.doi.org/10.4067/S0718-48832013000100008 Venegas, R.; S. Zamora; A. Galdames (2016). Hacia un modelo retórico-discursivo del macrogénero trabajo final de grado en licenciatura. Revista Signos, 49(Supl. 1), 247-279.

http://dx.doi.org/10.4067/S0718-09342016000400012 